\title{
MONUMENTOS FUNERARIOS PARA ANGELITOS EN EL CEMENTERIO GENERAL DEL SUR. UNA VISIÓN MEMORIAL SOBRE LA MUERTE
} FUNERARY MONUMENTS TO CHILDREN IN THE SOUTHERN GENERAL CEMETERY IN CARACAS, VENEZUELA.

\section{A MEMORIAL VISION OF DEATH}

Enviado em 12 de março de 2015 Aceito em 26 de abril de 2015

Jenny González Muñoz ${ }^{1}$

\begin{abstract}
Resumen: El presente artículo tiene por finalidad analizar la forma de ver la muerte $y$, por ende la vida, de la sociedad caraqueña, por medio de un acercamiento interpretativo a tumbas de infantes localizados en el Cementerio General del Sur (Venezuela), y cómo estos "lugares de memoria" son soportes de esa parte de la historia. En este sentido, se habla de "angelitos", forma coloquial de llamar a los pequeños fallecidos, quienes son enterrados en urna blanca, y cuya tumba es construida con atuendos de distintos materiales, adornada o diseñada con figuras de ángeles, juguetes y demás. La investigación, aún en proceso toma, como punto inicial cinco tumbas, que permitirán establecer un desarrollo hermenéutico que abarca tanto lo material como lo inmaterial, es decir, lo simbólico, la memoria familiar o colectiva. La metodología cualitativa es documental, método hermenéutico, por interpretación de fenómenos socioculturales.
\end{abstract}

Palabras clave: Tumbas para angelitos. Cementerio General del Sur de Caracas. Visión memorial.

\begin{abstract}
This article analyzes perceptions of death, and consequently, of life as well, within the society of Caracas, Venezuela. This analysis is carried out via an interpretive examination of children's graves in the Southern General Cemetery of that city, and the way that these "places of memory" are elements of that part of history. The colloquial term for these deceased children are "little angels". They are buried in white urns and their graves are constructed with
\end{abstract}

\footnotetext{
1 Licenciada en Artes por la Universidad Central de Venezuela. Magíster en Memoria Social y Patrimonio Cultural por la Universidad Federal de Pelotas (Brasil). Doctora en Cultura y Arte para América Latina y EI Caribe por la Universidad Pedagógica Experimental Libertador (Venezuela). Postdoctorado en Seguridad de la Nación por la Universidad Militar Bolivariana de Venezuela (actual). Docente y coordinadora del Doctorado en Patrimonio Cultural de la Universidad Latinoamericana y del Caribe (ULAC), sede Venezuela. E-mail: jenny66m@gmail.com
} 
decorations made of various materials, as well as adorned with representations of angels, toys, etc. The research project, still in progress, initially focusses on five graves, selected to be able to establish hermeneutical development that encompasses material and the immaterial elements, which is to say, symbolism, and family and collective memory. The qualitative methodology is documentary, hermeneutic, and carried out via interpretation of sociocultural phenomena.

Keywords: Children's graves. Southern General Cemetery of Caracas. Memorial vision.

\section{ENTONCES SE LEVANTÓ UN CLAMOR}

En su cuento El vampiro, el uruguayo Horacio Quiroga, expresa: "Son estas líneas las últimas que escribo. Hace un instante acabo de sorprender en los médicos miradas significativas sobre mi estado: la extrema depresión nerviosa en que yazgo llega conmigo a su fin" (2010, p. 376); se ha de saber que toda su escritura tiene por lei motiv la muerte, como una presencia tácita que se presenta en cada ser sin distinciones de ningún tipo. Dicha constante se intensifica al emanar de la propia vida del autor, quien incluso termina con su propia existencia en un acto más que suicida, inmortal.

Muchos individuos y pueblos, entre ellos los indígenas precolombinos del $A b y a$ Yala ${ }^{2}$, históricamente han visto la muerte como un paso más de la vida en el que luego de los rituales y ofrendas del caso, según el status social del difunto y demás elementos, el cuerpo era embalsamado o momificado y colocado en monumentos construidos específicamente para tal fin; los incas, por ejemplo, diseñaban una pirámide para cada rey como "morada" para la momia, la cual era colocada en el interior de la edificación junto con alimentos que, según, la creencia popular, servirían para alivianar el largo tránsito del alma hacia la eternidad (el alcanzar al Padre Sol Inti) y las esposas que deseaban quedarse con el fallecido, como una manera de demostrar amor y solidaridad eternos. Entonces, cada vez que era inminente la muerte de un rey inca se iniciaba la construcción de un monumento funerario, un memorial en su nombre, que guardaba su cuerpo, su espíritu (durante un tiempo) y sobre todo, perpetuaba su recuerdo en el colectivo.

\footnotetext{
2 Palabra de origen karibe-kuna que puede traducirse como "continente en expansión" y que se refiere a la extensión geográfica que va desde México hasta la Patagonia.
} 
Cuando una persona o grupo erige un monumento funerario lo que hace es extender la memoria del ausente más allá de la propia vida. Es decir, el recuerdo del difunto perdura más allá de la vida de sus contemporáneos, y es por eso que el monumento simboliza la prolongación de la vida (VÁZQUEZ; CORRAL, 2004, p. 19).

Como bien señalan Eduardo Vázquez y Adriana Corral (2004) el cementerio y sus monumentos, son reflejo de la cultura a la que pertenecen, en este sentido, hay que tomar en cuenta que tanto uno como el otro han sido construidos para que los vivos honren la memoria de sus difuntos, lo que plantea, a su vez, una doble acepción: por una parte, el homenaje al fallecido y, por otra, un "lugar de memoria" que funge como soporte para no olvidar a la persona que yace allí en la tumba. Pierre Nora parte del supuesto de la llegada de una suerte de inexistencia de la memoria en la contemporaneidad, para explicar que el ser humano ha creado los soportes intentando evitar el olvido, de modo que la "memoria es vista como una metáfora ya que el pasado está muerto, el futuro aún no llega y el presente se torna pasado en cuanto siquiera es terminado de nombrar" (GONZÁLEZ MUÑOZ, 2013, p. 125), el teórico francés observa al pasado como "a percepção global de qualquer coisa como desaparecida - uma ruptura de equilibrio" (NORA, 1984, p. 7). En este caso, cabe destacar que el autor se refiere específicamente a objetos como estatuas conmemorativas de hechos o personajes históricos, museos, placas, esculturas, pinturas, entre otros, lo cual, no obstante, también puede ser aplicado a las tumbas y los monumentos funerarios, porque son una suerte de "objetos simbólicos de la memoria, lugares donde se cristaliza y refugia la memoria" (LÓPEZ, 2011, p. 130).

Retomando la historia precolombina en materia fúnebre, se puede aseverar que como consecuencia de la evangelización de los indígenas, se introduce el bautismo para niños pequeños, quienes a la hora de morir (por enfermedad o cualquier otra causa) debían tener dicho sacramento para alcanzar la gloria, porque aunque eran pequeños llevaban el "pecado original", y para convertirse en "angelitos" debían ser expiados. Entonces, se van dejando de lado paulatinamente las tradicionales prácticas de colocar el cuerpecito momificado en jarras de arcilla, para dar paso a la urna y el entierro en cementerios antecedido de misa o rezos del catolicismo.

Todo este proceso relacionado con la muerte contribuyó para una dinamización de la concepción y visión de ésta, dando paso a una nueva postura más relacionada con las creencias religiosas con la naturalidad biológica propia de lo que nace, crece, se reproduce y muere, siendo el cementerio el lugar sin ecua non para albergar los 
cadáveres y los monumentos necesarios para afianzar la memoria respecto a los fallecidos (tanto para aquellos que los conocieron como para los que no ${ }^{3}$ ) y honrarlos.

La investigación de la que se devela el presente artículo se focaliza en un análisis de varias tumbas y sus atuendos funerarios dedicados a "angelitos" localizados en el Cementerio General del Sur de la ciudad de Caracas, Venezuela, con la finalidad de conocer cómo es concebido o visto el hecho de la muerte, desde lo memorial y la honra al difunto, en épocas de las primeras décadas del siglo XX y la contemporaneidad.

En primer lugar se hará un breve esbozo sobre las visiones de la muerte, luego se dará paso a un análisis explicativo sobre la historia del Cementerio General del Sur, con la finalidad de situar al lector en espacios y épocas pertinentes, para luego irse adentrando en el tema en cuestión por medio de la indagación en aspectos como los atuendos ${ }^{5}$ funerarios, la simbolización, haciendo hincapié en los infantes, mostrando, además, fotografías de algunos monumentos que ilustran parte de lo referido, siendo estos los lugares de memoria que aún se conservan.

\section{LA MUERTE: ¿TRÁNSITO O FIN DE UN PROCESO NATURAL?}

Phillipe Aries, en su libro El hombre ante la muerte hace varios acercamientos ontológicos sobre ésta desde perspectivas literarias e históricas a lo largo de los siglos. En este sentido, en el siglo XIX en Europa:

A pesar de su familiaridad con la muerte, los antiguos tenían la vecindad de los muertos y los mantenían aparte. Honraban las sepulturas, en parte porque temían el regreso de los muertos, y el culto que consagraban a las tumbas y a los manes tenía por objeto impedir a los difuntos 'volver' para perturbar a los vivos. Los muertos enterrados 0 incinerados eran impuros: demasiado cerca, amenazaban con mancillar a los vivos. La morada de los unos debía estar separada del dominio de los otros a fin de evitar cualquier contacto, salvo los días de los sacrificios propiciatorios (ARIES, 1984, p. 33).

Porque la muerte es algo cotidiano, inevitable, tal como dice en su primer capítulo "Todos vamos a morir", a pesar de ello, de acuerdo, por supuesto, al sistema

\footnotetext{
${ }^{3}$ En este caso se refiere a que personas ajenas al ver los monumentos de la tumba, se pueden acercar, leer el epitafio, tomar fotos, etc., y eso es también una manera de honrar, aunque indirectamente.

4 Apelativo utilizado cotidianamente en Venezuela para designar a los niños o niñas fallecidos, comprendiendo una edad aproximada entre 0 y 10 años.

5 Se ha preferido en este artículo hablar de "atuendos" para referirse a los monumentos o esculturas, puesto que lo hallado no son solo estos como tal, sino dibujos, grafitis, globos, etc.
} 
de creencias o la cultura de cada pueblo, ésta es tomada prácticamente como un tabú, puesto que se le teme $y$, por ende, se respeta. Los muertos, son colocados en lugares especiales, apartados y consagrados solo a los ceremoniales, se van construyendo "lugares de memoria" que promueven el recuerdo de fallecidos en la constancia de los vivos. Para el cristianismo de la época el ser incinerado era considerado impío, lo cual ha cambiado con el pasar de los años, tal vez por causa de la falta de espacio, sobre todo en las áreas urbanas. ${ }^{6}$ El temor a un posible regreso de los difuntos obligaba a construirles una suerte de "morada" en otros espacios, pero con acceso en libertad.

Los estoicos basaban la felicidad en la posesión de un alma libre, a la que no alcanza el dolor [...] Según ellos un espíritu anima al mundo y está presente en todas partes, las almas humanas sería sus emanaciones. Al morir el hombre se reuniría con este espíritu universal. La muerte sería un bien en sí, si no fuere porque para el sabio, que no es conquistado ni por el placer ni por la desgracia, no hubiera más grandeza que considerarla indiferente. Por tanto, se aceptaba la muerte y los valores como la dignidad y sobriedad inclinaban a aceptarla con naturalidad (ÁLVAREZ CHICANO, s. d., p. 13).

Según Metchnikoff (apud HERNÁNDEZ ARELLANO, 2006) "la angustia que genera la muerte se debe a que muy poca gente alcanza el fin normal de su existencia" $(2006$, p. 3) puesto que lo lógico es tener el desenlace en la vejez, pero cuando es a corta edad el choque psicológico es bastante fuerte, "no es natural". No obstante, como proceso inevitable, el fenecer tiende a ser consagrado por causas fundamentales, entre ellas, la negación a la partida sempiterna, una suerte de continuidad en el plano terrenal y la no aceptación de un posible olvido hacia esa persona, es por ello que se le consagra un lugar para recordarla de alguna forma, en la que el erigir monumentos, llevar flores o alimentos, prender velas, rendir tributo (religioso, político, social, etc.), es fundamental para mantener "viva la memoria" del fallecido.

El sistema de creencias mucho ha tenido que ver, tanto con la concepción de la muerte como con la manera de aceptarla o negarla. En este sentido, mientras el hinduismo promueve la reencarnación, siendo la muerte un proceso de tránsito del alma, donde el cuerpo físico es solo un vehículo para hacer posible la circulación en el samsara, religiones como las africanas (vudú, por ejemplo) rinden un culto a los muertos, pues es en el espíritu de sus familiares y tutores donde está la fuerza que acompaña a los vivos para cuidarlos y protegerlos; el cristianismo, por su parte, plantea una visión de la muerte desde la culpa y el sacrificio (ejemplo Jesús) siendo observada con miedo y sufrimiento.

${ }^{6}$ Cabe destacar en este particular que nos referimos exclusivamente a las culturas católicas o cristianas occidentales u occidentalizadas, puesto que hay otras realidades en este particular. 
Pero quizá ese "miedo" se deba fundamentalmente a la incertidumbre, a lo desconocido: el alma sale del cuerpo y va a un lugar que el humano, por más que lo intente, aún no lo descifra y sigue siendo una total interrogante. Los que han cruzado esa frontera no han vuelto para contar nada y ese es un elemento esencial. El humano entonces, se instala en dos puntos paralelos: acrecentar el rol de tabú frente a su finitud y consagrar lugares para evitar el olvido. En este punto es conveniente preguntarse: ¿olvido de quién? ¿Se dedica esos lugares para albergar los difuntos, para olvidar la muerte o para tener presente su rol inevitable? ¿La tumba es lugar de memoria para el fallecido o para el vivo?

Tal como devela Flor Hernández Arellano (2006) es la cultura la que moldea las experiencias acerca de la pérdida y las ritualizaciones que se hagan en torno a ella, lo que obedece a patrones de conducta dinámicos según el contexto histórico de las diversas sociedades, donde puede ser desde una maldición o un castigo de dios, hasta el descanso eterno de un cuerpo golpeado, por ejemplo, por una penosa enfermedad. Frente a esto, en los pueblos venezolanos buena parte del siglo XX la persona fallecida era velada en la propia casa hasta que tenía "cristiana sepultura", previa misa a cuerpo presente en la iglesia de la localidad; esto se debe a la cercanía del fallecido y la negación de su partida para siempre, es el no retorno, la ausencia que puede conllevar al olvido. Esto ocurre en las sociedades occidentales u occidentalizadas, pues en muchas culturas indígenas la práctica del olvido hacia los muertos es interesante, por ejemplo, al no repetir el nombre de quien muere, dicha decisión está orientada a un respeto al tránsito. En estos casos pasar a "mejor vida" no es un mero eufemismo, sino algo posible desde lo intangible.

\section{LOS MONUMENTOS FUNERARIOS COMO LUGARES DE MEMORIA}

En Caracas, el 5 el julio ${ }^{7}$ de 1876 es inaugurado el Cementerio General del Sur (por su ubicación dentro de la capital), construido bajo la presidencia de Antonio Guzmán Blanco, quien se caracterizó en sus mandatos ${ }^{8}$ por la suntuosidad escultórica y arquitectónica, inspirada en sus viajes a Francia. Es puesto en funcionamiento cinco días después convirtiéndose en el único cementerio de la ciudad, acabando así con los cementerios particulares, especialmente en los lugares céntricos, quedando entonces eliminados por razones de higiene y estética. Según consta en la página electrónica

\footnotetext{
${ }^{7}$ Día en que se celebra la Firma del Acta de la Independencia, acaecida en 1811.

${ }^{8}$ Guzmán Blanco fue presidente de Venezuela en tres períodos: 1870 - 1877, 1879 - 1884, y 1886 - 1887. Era apodado "El llustre Americano".
} 
oficial de la Fundación Alcaldía de Caracas, éste se hizo para abrigar las necesidades de la población de ese momento y:

(...) conjuntamente con el general (sic) Lirio Duarte Level, escogieron los terrenos denominados "Tierra del Juego" ubicados en lo que se llamaba el Rincón de El Valle, bautizado más adelante como Prado de María.

Desde entonces el Cementerio General del Sur, cuenta entre sus fortalezas con una variedad de servicios que presta como el de inhumaciones, exhumaciones, donaciones de fosas adultos $y$ pequeñas, solvencia de mantenimiento, verificaciones de linderos, así como el servicio funerario con capillas velatorias, para misas y en proyecto se encuentra el primer crematorio público nacional.

Este Campo Santo también es reconocido, pues en él reposan los restos de personajes célebres de la historia venezolana como: Armando Reverón ${ }^{9},(\ldots)$ Rómulo Gallegos ${ }^{10}$, Andrés Eloy Blanco ${ }^{11}$, Aquiles Nazoa ${ }^{12}$, Fabricio Ojeda ${ }^{13}$, Miguel Otero Silva ${ }^{14}$, entre otros.

De la misma forma, el Cementerio General del Sur, cuenta con grandes riquezas artísticas como "La Capilla del General Crespo", panteones como los del general (sic) Isaías Medina Angarita; Monumento de Emilio Fernández; Monumento "Mártires del 27F", en homenaje a los caídos del 27 de febrero de 1989; Los Caballotes, de los Bomberos, de la Policía Metropolitana, Guardia Nacional y del Instituto de Oficiales retirados de la Fuerza Armada. ${ }^{15}$

Además de los monumentos y panteones citados arriba, existe una cantidad significativa de aquellos dedicados a "angelitos", con representación de diversas escenas o figuras, dentro de las que se puede destacar niños orando, madres llorando, corazones y sobre todo, ángeles. Es de destacar que en las primeras décadas del siglo XX se está consolidando una nueva etapa de la humanidad, ya que es tiempo de grandes acontecimientos como la invención del automóvil, el posicionamiento político de Japón como consecuencia de su triunfo en la guerra contra Rusia, se desarrolla la

\footnotetext{
${ }_{9}^{9}$ Artista plástico venezolano (1889-1954) de alta relevancia nacional. Considerado por muchos como el mejor del siglo XX.

10 Uno de los más renombrados novelas venezolanos (1884-1969). Además de educador y escritor fue político, llegando a la presidencia de la República en 1948 por un corto período de nueve meses, acabando por ser derrocado.

11 Poeta venezolano (1896-1955). Su poema "Píntame angelitos negros" es considerado de alta relevancia en la nueva manera de abordar temas de discriminación en la literatura de su tiempo.

${ }^{12}$ Escritor, periodista, poeta y humorista venezolano (1920-1976).

13 Conocido luchador social comunista y periodista venezolano (1929-1966).

${ }^{14}$ Escritor y periodista venezolano (1908-1985). Autor de la famosa novela Casas muertas.

${ }^{15}$ Acceso: $\quad<h t t p: / / w w w . f u n d a c a r a c a s . g o b . v e /$ ?module=pages\&op=displaypage\&page_id=38\&format $=$ html>. Consulta en: 30 sep. 2013.
} 
Primera Guerra Mundial, se lleva a cabo el primer vuelo con avión a motor, es tiempo de la Revolución Mexicana. Mientras que Venezuela ha salido de la opulencia monumental de Antonio Guzmán Blanco, lo cual se ve reflejado en los memoriales funerarios, a pesar de que es un país de poco desarrollo económico, basado fundamentalmente en la agricultura y ganadería, y bajo una dictadura ${ }^{16}$, el Cementerio General del Sur es un lugar donde se ve la marcada diferencia social de los habitantes de la capital, precisamente por causa de las distintas construcciones en las tumbas.

Figura 1 - Panteón familiar con monumento que muestra al ángel frente a la cruz, simbolizando la paz del reino de Cristo. Se observa el detalle del encaje en el cuello y parte baja de la falda del traje, la flor en la mano, columnas corintias, guirnalda. La fecha de defunción es 1920. Cementerio General del Sur - Caracas

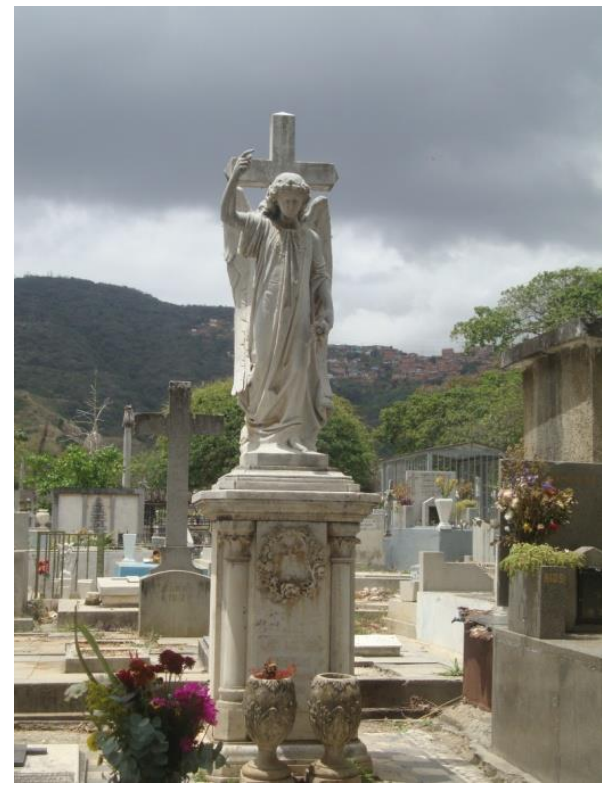

Fonte: Foto acervo de la autora.

Durante la presidencia de Guzmán Blanco, Venezuela experimenta grandes avances, destacando servicio de agua potable, inicio del alumbrado eléctrico, construcción de bulevares, teatros, plazas, muestras escultóricas, obras arquitectónicas, coches a caballo, el ferrocarril, en fin, obras que modifican el comportamiento de la sociedad del momento por medio de un "afrancesamiento" pretendido por el gobernante, insertado desde el arte. Dentro de ese esplendor se crea el Cementerio General del Sur. Espacio que se convierte en "lugar de descanso" de los fallecidos caraqueños, donde la diferenciación socio-económica se manifiesta en la presencia de obras escultóricas mandadas a hacer a artistas a gusto de la familia, según la personalidad del difunto, poseyendo copias de obras de diversos países europeos como Italia y Francia (RODRÍGUEZ, 2011).

${ }^{16}$ Entre 1899 y 1908 gobierna Cipriano Castro; y entre 1908-1935 está al mando Juan Vicente Gómez. 
El camposanto llegará a ser pues, un lugar en donde se presentan obras de arte que a pesar de ser privadas pueden ser disfrutadas por el colectivo. Necrópolis, jardín o museo, la morada final de los difuntos caraqueños exaltaría a través de la belleza de los panteones el lugar de descanso de las familias más pudientes, quienes comprarán lotes de terreno para asegurar la colocación de obras de arte en los lugares de descanso de sus familiares (RODRÍGUEZ, 2011, p. 188).

También la diferenciación se ve en el traslado de los difuntos, siendo en carreta para los pudientes y en hombro de familiares y/o amigos para los menos acaudalados. Es interesante en este punto señalar que se guardaba luto en la vestimenta, siendo negro, blanco o gris los tonos a utilizar, variando el tiempo de uso según la filiación con el difunto, y en las casas se colocaba cortina blanca en la puerta amarrada con una cinta gruesa de color morado oscuro. En lo que se refiere al fallecimiento de infantes no se usaba el luto rígido negro, pero si un respeto a la memoria y ausencia demostrado con colores claros, siendo la urna de color blanco. Con la construcción del Cementerio General del Sur, de características ya señaladas, la visión de la muerte desde el memorial se transforma en los caraqueños,

sobre todo en aquellas familias de mayores recursos (en la forma de vestir, en la forma de decorar los panteones) sin descartar al ciudadano común que también utilizaba las instalaciones del camposanto no sólo para visitar a su deudo, sino también para la contemplación de las obras de arte que en este se encuentran (RODRÍGUEZ, 2011, p. 191-192).

Cabe destacar que para dicha época la ciudad de Caracas es menor a la actual, tanto en densidad como en espacio territorial, de modo que este cementerio quedaba en las afueras, con fácil acceso por medio del ferrocarril; lo que muda como consecuencia de la expansión de la capital, llegando a estar (tal como hoy día) dentro de una urbanización, ésta de clase social de pocos recursos económicos que, por cierto, también se llama El Cementerio, de modo que los habitantes comúnmente, y a modo de broma, suelen exclamar: "vivo en El Cementerio, pero de los vivos", juego de palabras que apunta a una re-significación de la muerte y sus lugares de memoria.

El Cementerio General del Sur, a pesar de que aún hoy día, en 2015, comparte acciones con el Cementerio del Este, cuya conformación no permite erigir ningún tipo de monumento, aún es utilizado, sobre todo por gente de poder adquisitivo escaso, no perdiendo su vigencia, aunque hay que recalcar el franco deterioro que ha ido acumulando durante años, producto de una importante desidia colectiva. A pesar de esto, por causa de su historia y las verdaderas obras de arte que alberga, en 1982, es declarado Monumento Histórico Nacional. 
Desde el día de la apertura de sus puertas ha pasado por mudanzas lógicas de todo proceso histórico-cultural, no obstante, continúa siendo "lugar de memoria" para los vivos que desean perpetuar el recuerdo de sus muertos (en este caso particular de investigación en lo referente a niños) y como memoriales que los honran visiblemente ante los demás ajenos.

\section{MONUMENTOS PARA ANGELITOS: LUGARES POR NO OLVIDAR}

Ya desde la Edad Media la tumba representa "la casa del muerto" (Azara, 1999), de allí que sea vista como la morada final; el cuerpo yace en ese espacio entre la tierra y la madera, volviendo a ser polvo, como dicen los textos bíblicos, consiguiendo su sustancia esencial. En la construcción de la tumba y sus características se refleja las vivencias de cada sociedad, sus fortalezas y debilidades, sus creencias y la actitud frente al deceso.

Existen distintos tipos de pérdidas, como la muerte de un hijo, un dolor que ha sido conceptualizado como uno de los más profundos y difíciles de resolver en el tiempo, debido a las fuertes reacciones emociones que dicho suceso despierta y provoca en los progenitores. Si la pérdida es súbita e inesperada, como cuando es producto de una enfermedad terminal en el niño, el tránsito del duelo es más difícil (YOFFE, 2009, p. 132).

Es el apego uno de los sentimientos más relevantes del luto y eso se manifiesta, frente a la muerte prematura del humano, de diferentes maneras de establecer la activación de la memoria, por ejemplo la "celebración" del cumpleaños del infante difunto con globos, música, incluso torta, en la propia tumba, siendo un acto totalmente ritual de negación a la partida sempiterna, al no retorno. Dicha ritualización la hemos visto personalmente en algunas visitas al cementerio lugar de la presente investigación. Asimismo, se ha podido observar tumbas de ángeles con gesto de tristeza y resignación coloreados de tonos pasteles. 
Figura 2 - Tumba de infante en la que no se puede conocer la edad de muerte del "angelito", pero por las características se devela que era pequeño. En el epitafio se lee: "Eddy Alexander Barón Jiménez + 29-6-94 Rdo de sus padres y familiares".

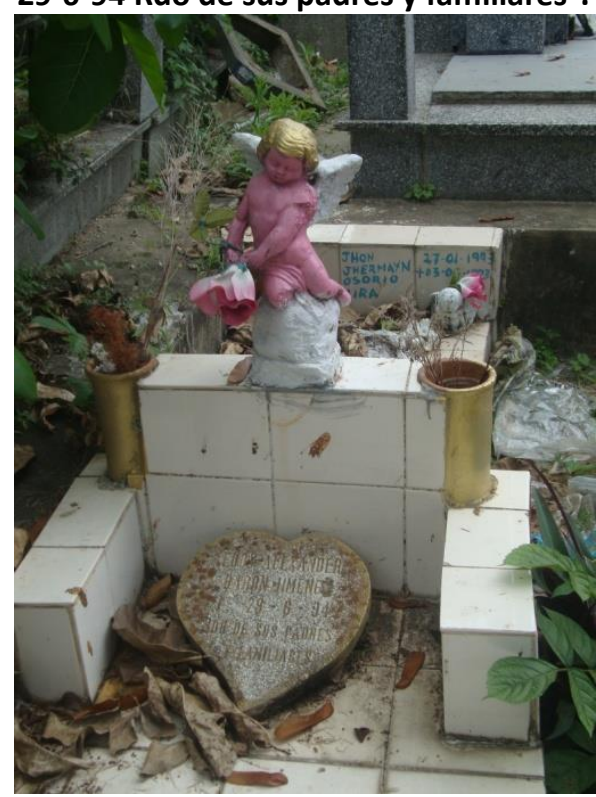

Fonte: Acervo de la autora.

Tal como se observa en la imagen anterior, la investigación ha arrojado dificultad para conocer la edad de muerte de los infantes, puesto que en muchas de ellas solo se refleja la fecha de deceso y no de nacimiento, a lo que hay que agregar el alto grado de deterioro que tienen muchas de ellas debido al abandono del camposanto en líneas globales. Se ha visto como elemento común el corazón y el ángel (juntos o no) en las tumbas de infantes, cambiando tanto diseños como materiales de construcción de acuerdo a la época. Cabe destacar, en relación a la figura de arriba, que la pintura del ángel (rosado en el cuerpo, alas blancas y dorado en el cabello) es posterior a la original, de igual modo, la flor de plástico colocada en las manos del mismo.

Becker acota sobre los ángeles:

La noción de una corte celestial o consejo que asiste al Dios de Israel se abre paso tardíamente (todavía los ángeles del arte paleocristiano carecen de nimbo y de alas, siendo más parecidos a la idea judía de unos simples mensajeros de Dios, que es como los describe el Antiguo Testamento) por influencia de los zoroástricos y de la literatura apocalíptica de los ss. I y II, que desarrollan la particular función de los ángeles como mediadores entre los reinos de este mundo y el Reino de Dios; en el s. $V$ el pseudo-Dionisio estableció las tres categorías angélicas, cada una de las cuales se divide, a su vez, en tres coros: $1^{\circ}$ serafines, querubines y tronos; $2^{\circ}$ dominaciones, virtudes y potestades; $3^{\circ}$ principados, arcángeles y ángeles (p. 30). 
Una de las pocas tumbas encontradas con especificación de fecha de nacimiento y muerte es la de Ana María Amadis Rosario (20-7-81 + 8-2-85). Es una lápida de diseño limpio cuyo único atavío es la foto de la niña fallecida, de vestido sentada en una silla.

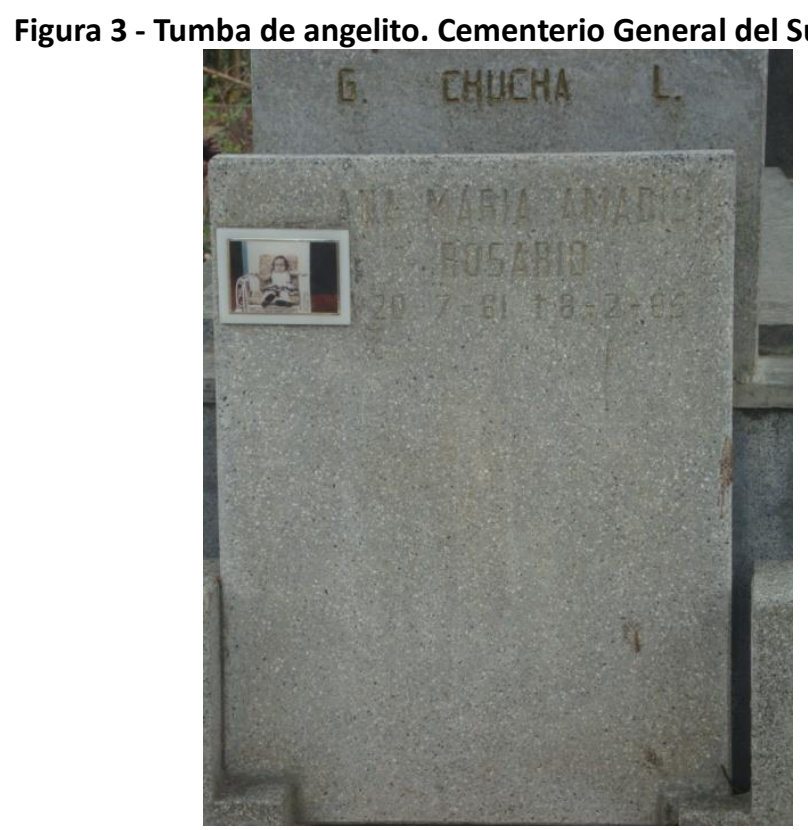

Fonte: Acervo de la autora.

Como modo de resguardo el cuerpo fallecido suele tener, en ocasiones, un doble encierro: la lápida con sus atavíos y las rejas que las convierten en una suerte de morada construida para aislarlo del entorno que lo rodea. La tumba que se presenta más adelante está construida con obra minimalista, pero se ha colocado para protección, rejas y techo, de manera que la posibilidad de la profanación es disminuida. Es interesante las plantas sembradas, es decir, no hay floreros de uso inmediato; se observa el corazón grande con el epitafio, al pie del pedestal el libro de recuerdo de sus tíos, y a los lados el recuerdo de sus abuelos y hermano. La leyenda destaca que el "angelito" José Emmanuel nació y murió en pocas horas, a pesar de que han pasado 26 años de su fallecimiento, la tumba está en perfectas condiciones, revelando así que este lugar de memoria sirve de apego a los familiares que mantienen vivo el recuerdo de alguien cuya existencia fue muy breve. 
Figura 4 - Tumba resguardada con rejas. Destaca el corazón y libro de recuerdos.

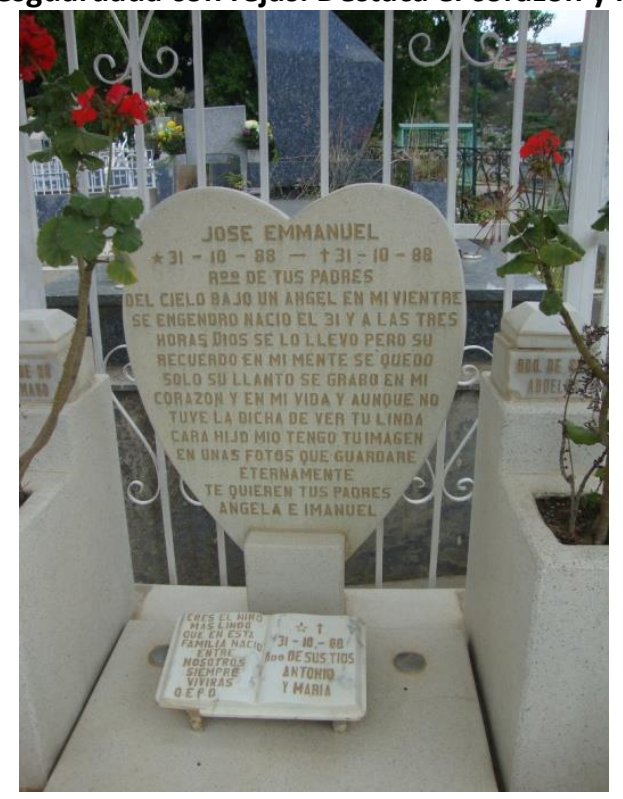

Fonte: Acervo de la autora.

Otra tumba encontrada fue la de Samira Femimal Castillo Zambrano, "angelito" de reciente data (3-4-2013 + 18-4-2013); el lugar presenta buen estado de conservación con incorporación de flores artificiales, lo cual se supone que para evitar el deterioro de las naturales no permitiendo afectar la estética del memorial, también destaca la figura de la muñequita hecha en foami, pintada a mano y recortada, además de las piedritas de color rosado colocadas en la parte superior de la lápida. De estos atavíos se puede inferir la capacidad económica de la familia del "angelito" que yace allí, quienes al estar imposibilitados de colocar monumentos y resguardos costosos, la han decorado de modo "casero". Al visitar ese espacio nos pareció una especie de flor de loto, puesto que todo alrededor era destrucción y sucio.

Figura 5 - Tumba de "angelito" con atavíos manuales, destaca el corazón con el epitafio.

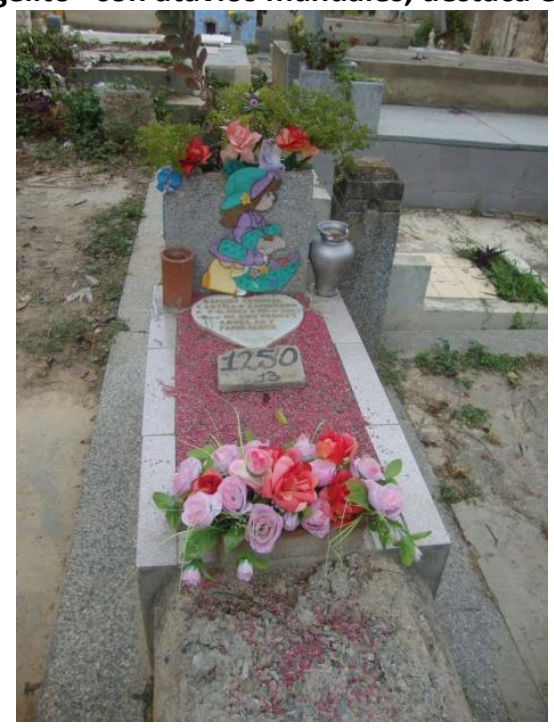

Fonte: Acervo de la autora. 
Por último se presenta una lápida que carece de sentido de la arquitectura, lo cual hace pensar en la posibilidad de una configuración familiar, donde destaca jardineras, columnas pequeñas para colocar velas $u$ otros ofertorios, un banco para sentarse frente a la lápida. Importante la no presencia de la cruz, salvo la pintada en la parte principal debajo del nombre. Se lee: "José Fidel Romero Fariñe 24-5-86 + 13-1288 Rdo de sus padres" y se distingue una jardinera con plantas naturales, entre ellas la sábila (planta que simboliza la buena suerte), un frasco de compota volteado con pétalos en su interior, flores recortadas y mariposas en el suelo, flores hechas a mano colocadas en botellas de refresco, angelitos blancos y negros hechos de foami suspendidos de una cuerda, un ángel de foami introducido en la jardinera principal; todo lo cual lleva a pensar que recientemente hubo en esa tumba una conmemoración que quisieron compartir con el "angelito" difunto.

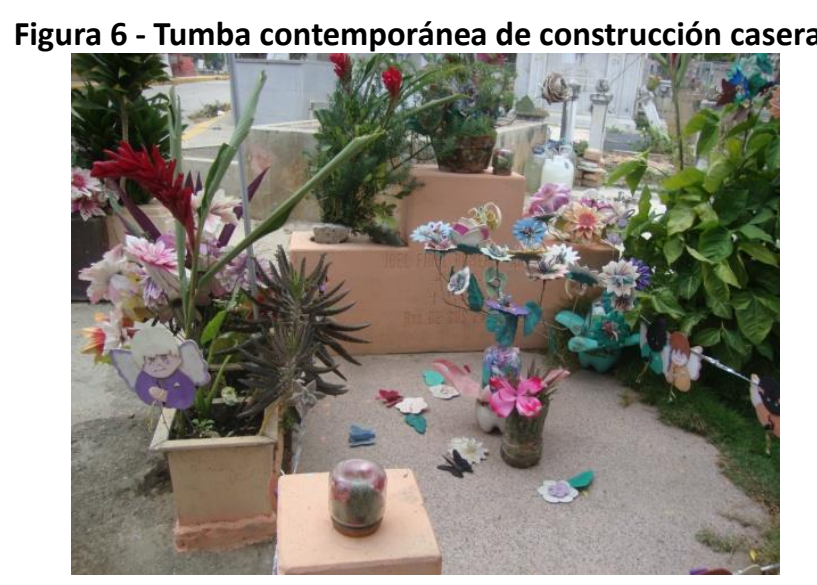

Las tumbas estudiadas tienen un lenguaje simbólico a través del cual se puede descifrar el cambio de aproximaciones hacia la muerte como un hecho que se venera, de una u otra manera, por medio de los lugares de memoria que se desarrollan en los cementerios. Siendo este proceso natural violentado con el deceso de infantes de poca edad, donde lo lógico es que los hijos entierren a los padres y no lo contrario, las lápidas de "angelitos" y todo lo que ellas develan, crean un paisaje cultural capaz de ser abordado a través de la alegoría para dar testimonio de épocas y procesos históricos de la Caracas del siglo XX y XXI, conllevando a pensar en la reconstrucción de la memoria histórica de los habitantes de la ciudad desde lo inexorable de la muerte.

\section{A MODO DE EPÍLOGO}

La Caracas de los gobiernos de Antonio Guzmán Blanco configuró una ciudad reflejo de una sociedad guiada por el "progreso", donde el furor de arquitectura y arte opulentos marcaron el camino de toda la existencia desde el nacimiento hasta la 
muerte misma. Partiendo de los cementerios como lugares de memoria que sirven para recordar a los muertos y evitar el olvido en los vivos, se ha conseguido ver que sobre todo en las primeras décadas del siglo pasado era común la utilización de grandes monumentos, esculturas, rimbombantes panteones, mausoleos consagrados a la ritualización para los seres queridos, por supuesto en familias con alto poder adquisitivo, lo cual ha ido cambiando con el pasar del tiempo, de suerte que en el siglo XXI se aplaca la monumentalidad, dando paso a tumbas minimalistas que, por medio de un solo elemento simbólico, por ejemplo, un corazón o un ángel, siendo el primero el amor interminable y profundo, mientras el segundo la pureza, se puede rendir culto con los mismos resultados respecto al recuerdo.

En el Cementerio General del Sur de Caracas se desarrolla un paisaje cultural en el que confluyen diversos estratos de la historia y clases socio-económicas que marcaron un esplendor nacido a finales del siglo XIX, que cobró gran aplomo en las primeras décadas del siglo XX, llegando a un declive que en la actualidad se observa en el deterioro de sus tumbas y monumentos funerarios. Respecto a las tumbas de "angelitos" se ha visto el mismo caso de suntuosidad escultórica en ciertos momentos para contemporáneamente, llegar a lápidas con atavíos minimalistas, pero quizá más sentidos, como son muñecos pintados por los mismos familiares.

Cada tumba de infante es un lugar de recuerdo, mantiene un apego donde la esperanza se mezcla con la melancolía; en muchos memoriales del siglo pasado solo se coloca la fecha de fallecimiento, pero la configuración de elementos que atavían la tumba dan una idea clara de que pertenece a un "angelito"; no hay golondrinas, que simbolicen la resurrección, sino elementos gustados por cualquier niño, colores utilizados para infantes. Algunas tienen solo los datos, otras un epitafio largo y sentido que amplía el soporte para recordar en el tiempo, mas tal como se planteó en el cuerpo de este texto, estos son lugares consagrados ¿a la memoria de los vivos o de los muertos?

\section{REFERENCIAS}

ÁLVAREZ CHICANO, Carlos. Las diferentes concepciones de la muerte en las principales culturas de la humanidad: origen de su significación en la sociedad actual. [19_]. Disponible en: <http://www.cartapacio.edu.ar/ojs/index.php/byb/article/viewFile/264/168>. Consulta en: 10 oct. 2013.

ARIES, Phillipe. El hombre ante la muerte. Madrid: Taurus, 1984. 
AZARA, Pedro. La casa de los muertos (sobre tumbas modernas). In: GIL, Mónica (ed.) La última casa. Barcelona: Gustavo Gilli, 1999. p. 8-23.

BECKER, Udo. Enciclopedia de los símbolos. México: Océano, 2001.

GONZÁLEZ MUÑOZ, Jenny; MAZZUCCHI FERREIRA, Maria Leticia. Conflictos y dilemas en la construcción de los lugares de memoria. IN: GONZÁLEZ MUÑOZ, Jenny (coord.) Memorias, sociedades, políticas y procesos culturales de América Latina. Caracas: UNESR, 2013. p. 117139.

HERNÁNDEZ ARELLANO, Flor. El significado de la muerte. Revista Digital Universitaria, México, vol. 7, n. 8, 2006. Disponible en: <http://www.revista.unam.mx/vol.7/num8/art66/ago_ art66.pdf>. Consulta en: 15 agos. 2013.

LOPEZ, Derechos humanos, patrimonio y memoria. Museos de memoria y sitios de conciencia. In: ERAZO; RAMÍREZ; SCANTLEBURY (eds.). Derechos humanos, pedagogía de la memoria y políticas culturales. Santiago: LOM, 2011. p. 127-138.

NORA, Pierre. Entre mémoire et historique: la problématique des lieux. In: NORA, Pierre (orgs.). Les lieux de mémoire: vol. 1: la Republique. Paris: Gallimard, 1984.

RODRÍGUEZ, Yuleima. Cementerio general del Sur: Aproximación histórica y costumbres de los caraqueños vistas a través de las necrópolis. In: Revista Mañongo, N 36, Vol XIX, 2011, pp- 173 - 198. Disponible en: http://servicio.bc.uc.edu.ve/postgrado/manongo36/art07.pdf. Consulta en: 30 sep. 2013.

VÁZQUEZ SALGUERO, David Eduardo; CORRAL BUSTOS, Adriana. Monumentos funerarios del Cementerio del Saucito: San Luis Potosí, 1889-1916. San Luis Potosí: El Colegio de San Luis, 2004.

YOFFE, Laura. El duelo por la muerte de un ser querido: creencias culturales y espirituales. In: Psicodebate 3. Psicología, Cultura y Sociedad, 2009. Disponible en: <http://www.palermo.edu/cienciassociales/publicaciones/pdf/Psico3/3Psico\%2009.pdf>. Consulta en: 22 sep. 2013. 